\title{
Polarization conversion and "focusing" of light propagating through a small chiral hole in a metallic screen
}

\author{
A. V. Krasavin, ${ }^{\text {a) }}$ A. S. Schwanecke, and N. I. Zheludev \\ EPSRC NanoPhotonics Portfolio Centre, School of Physics and Astronomy, University of Southampton, \\ SO17 1BJ, United Kingdom \\ M. Reichelt, T. Stroucken, and S. W. Koch \\ Department of Physics and Material Sciences Center, Philipps-University, Renthof 5, \\ D-35032 Marburg, Germany \\ E. M. Wright \\ Optical Sciences Center and Department of Physics, University of Arizona, Tucson, Arizona 85721
}

(Received 25 January 2005; accepted 12 April 2005; published online 10 May 2005)

\begin{abstract}
Propagation of light through a thin flat metallic screen containing a hole of twisted shape is sensitive to whether the incident wave is left or right circularly polarized. The transmitted light accrues a component with handedness opposite to the incident wave. The efficiency of polarization conversion depends on the mutual direction of the hole's twist and the incident light's wave polarization handedness and peaks at a wavelength close to the hole overall size. We also observed a strong transmitted field concentration at the center of the chiral hole when the handedness of the chiral hole and the wave's polarization state are the same. (C) 2005 American Institute of Physics.
\end{abstract}

[DOI: $10.1063 / 1.1925759$ ]

Metallic films structured on the nanoscale show a range of unusual properties, such as extraordinarily high transmission of light through metallic films perforated with round, rectangular and C-shaped holes, ${ }^{1-3}$ and strong linear birefringence for asymmetrical openings. ${ }^{4,5}$ Metallic films with arrays of chiral (twisted) holes, i.e., holes that are not their mirror images over any line in the plane of the film, are of special interest. They show polarization effects in diffraction that are enantiomer sensitive, i.e., depend on whether the structural elements of the array are twisted clockwise or anticlockwise, ${ }^{6}$ while their microscope images in polarized light display unusual symmetries. ${ }^{7}$ Here, we show that propagation of light through a metallic screen containing a hole of twisted shape is sensitive to whether the incident wave is left (LCP) or right circularly polarized (RCP). The transmitted light accrues a component with handedness opposite to the incident wave intensity which peaks at a wavelength close to the hole overall size. We also observed a strong concentration of the transmitted field at the center of the chiral hole when the handedness of the chiral hole and the wave's polarization state are the same.

For our study of chirality sensitive light propagation through a chiral hole, we took a chiral opening of gammadion shape as an example. The gammadion arms were bent at $45^{\circ}$, as shown in Fig. 1(a), to make a clockwise twisted structure, with a positive geometrical chirality index, as calculated according to the definition in Ref. 8. We define it as a right handed gammadion. The gammadion opening in the screen has four-fold rotational symmetry around an axis perpendicular to the screen and a plane of symmetry that is parallel to the screen. We investigated a free-standing flat gold film of thickness $d=140 \mathrm{~nm}$ with a single gammadion shaped slit cut into it. The width of the gammadion slit was chosen to be $w=0.6 \mu \mathrm{m}$, while the total length of the gam-

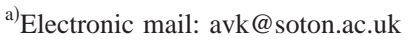

madion, measured from the end of one arm to the end of the opposing arm, was $L=4.1 \mu \mathrm{m}$ [Fig. 1(a)]. For our analysis, we used realistic values of the complex dielectric coefficient of gold at different wavelengths from Ref. 9. To evaluate the propagation of light through the metal screen with its chiral opening, we numerically modeled the electromagnetic fields at a distance of $160 \mathrm{~nm}$ from the screen on the opposite side to a normally incident plane monochromatic electromagnetic wave. In our analysis, we used the FEMLAB software package that implements a true three-dimensional finite element method for solving Maxwells equations. ${ }^{10}$ We studied the transmission of the chiral hole for light at wavelengths $\lambda$ from $1.3 \mu \mathrm{m}$ to $5.6 \mu \mathrm{m}$, thus covering wavelengths both shorter and longer than the characteristic size of the gammadion opening $L=4.1 \mu \mathrm{m}$. All results are presented in terms of the Stokes parameters of the incident $S_{i}^{\text {in }}$ and transmitted $S_{i}^{\text {tr }}$ fields that are defined in a standard fashion ${ }^{11,12}$ in a Cartesian coordinate frame. In this case, $S_{0}$ is proportional to the light intensity, while the combinations $\left(S_{0} \pm S_{3}\right) / 2$ represent the intensities of the left- and right-handed polarization components of the field.

We found that the light transmitted through the structure accrues a polarization component opposite to the polarization state of the incident wave, giving rise to a circular polarization conversion effect. Thus, the transmitted field becomes elliptically polarized, with its polarization state being a function of coordinate $\mathbf{r}$ in the $x y$ plane. Figure 1 illustrates a dramatic polarization conversion at $\lambda=4.1 \mu \mathrm{m}$. The observed polarization conversion effect with circularly polarized light is governed by chirality. It is different by its manifestation and nature from the well documented polarization conversion effect for linearly polarized light that is driven by anisotropy of the structure and may be seen in a metal grating consisting of a series of high and narrow ridges that are oriented at $45^{\circ}$ to the polarization angle. ${ }^{13}$ The dependence of the effect reported here on the handedness of a circularly polarized wave is obvious when comparing the intensity 


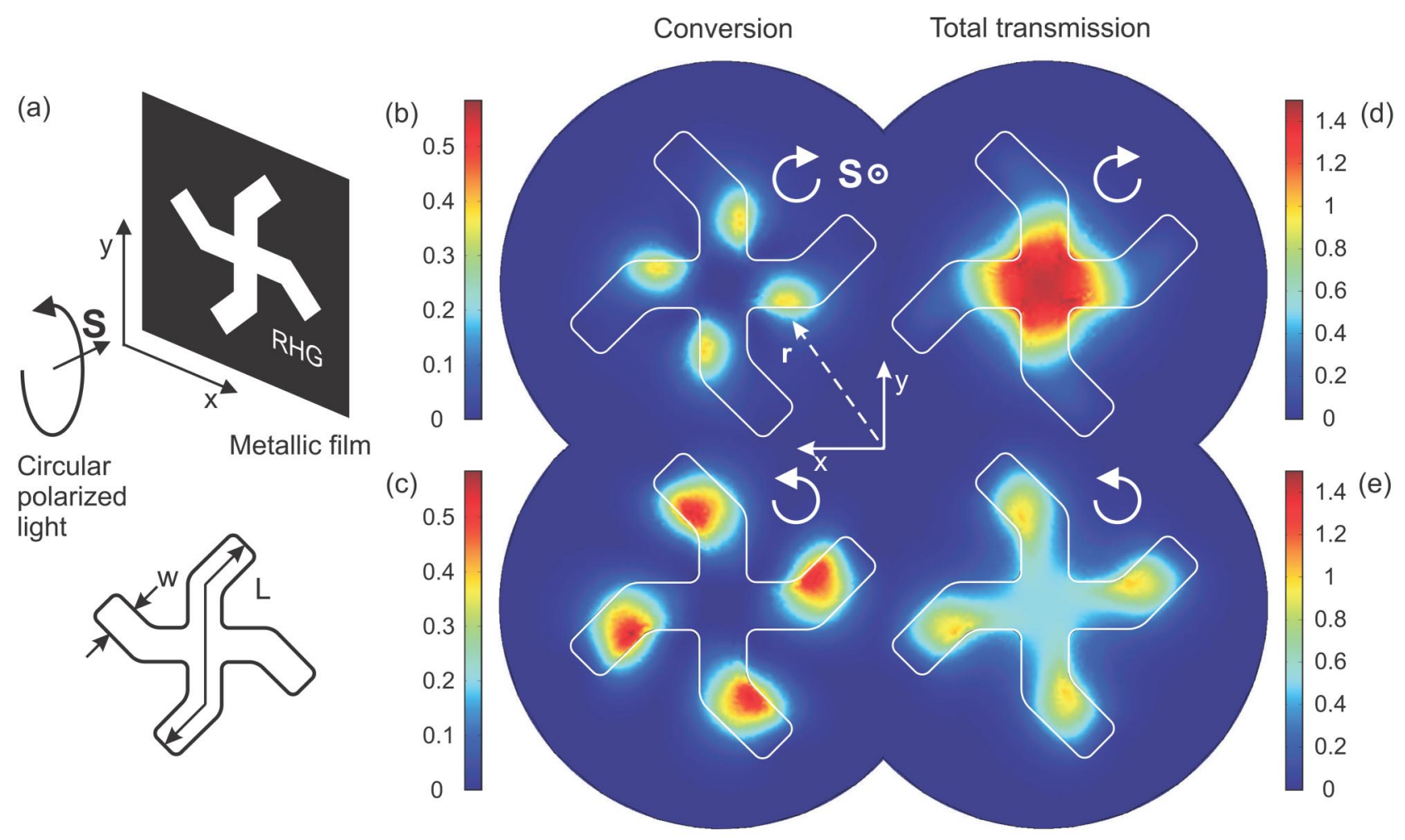

FIG. 1. (Color online) (a) General arrangement of the numerical simulations. Left field maps: Intensity $\left[S_{0}^{\mathrm{tr}}(\mathbf{r}) \pm S_{3}^{\mathrm{tr}}(\mathbf{r})\right] /\left[2 S_{0}^{\mathrm{in}}(\mathbf{r})\right]$ of the polarization converted component of transmitted radiation for (b) RCP and (c) LCP incident waves. Right field maps: Intensity $S_{0}^{\text {tr }}(\mathbf{r}) / S_{0}^{\text {in }}(\mathbf{r})$ of total transmitted radiation for (d) RCP and (e) LCP incident waves. For all field maps, the incident wave propagates in the direction of the reader. The arrows illustrate the rotational direction of the electric field vector of the incident wave in the plane of the structure.

maps of transmitted light with converted polarization for illumination with a RCP plane wave [Fig. 1(b)] and a LCP plane wave [Fig. 1(c)]. These field maps are dramatically different. The effect of enantiomeric sensitivity is also illustrated by Figs. 1(d) and 1(e), which show that total transmission is sensitive to the mutual handedness of wave and gammadion hole.

By integrating the intensities of the two circularly polarized components over the whole gammadion opening, we calculated the overall power transmission $T$ $=\int S_{0}^{\mathrm{tr}}(\mathbf{r}) d \sigma / \int S_{0}^{\mathrm{in}}(\mathbf{r}) d \sigma$ and the overall polarization conversion efficiency $K_{ \pm}=\int\left[S_{0}^{\mathrm{tr}}(\mathbf{r}) \pm S_{3}^{\mathrm{tr}}(\mathbf{r})\right] d \sigma / \int 2 S_{0}^{\mathrm{in}}(\mathbf{r}) d \sigma$ of the screen with chiral hole at different wavelengths (here, $d \sigma$ is an area element of the observation plane surface). The wavelength dependence of the overall power transmission of the hole [Fig. 2(a)] shows only a small variation with the wavelength. Within the accuracy of our numerical method, we found no dependence of the overall power transmission on the incident polarization state. However, chiral effects are very pronounced in polarization conversion, as can be seen from Fig. 2(b). When the handedness of the chiral hole and the wave are opposite [solid line on Fig. 2(b)], the polarization conversion efficiency reaches a maximum at a wavelength close to the characteristic size of the chiral opening, $\lambda \simeq L$. When handedness of the chiral hole and the wave coincide, the polarization conversion is nearly a factor of 2 less efficient and has a maximum shifted to a shorter wavelength [dashed line on Fig. 2(b)]. The chiral sensitivity diminishes for incident light wavelengths $\lambda$ much shorter than the characteristic size of the chiral opening $L$. This is demonstrated by the convergence of solid and dashed lines in Fig. 2(b) at $\lambda<1800 \mathrm{~nm}$. If these numerical experiments are Downloaded 15 May 2005 to 152.78.194.84. Redistribution subject repeated with a left-handed opening (a mirror image of the structure in Fig. 1), the polarization conversion effect becomes more efficient for right-handed circularly polarized light. From here, one can make the intriguing observation that since the perceived sense of twist of the chiral hole

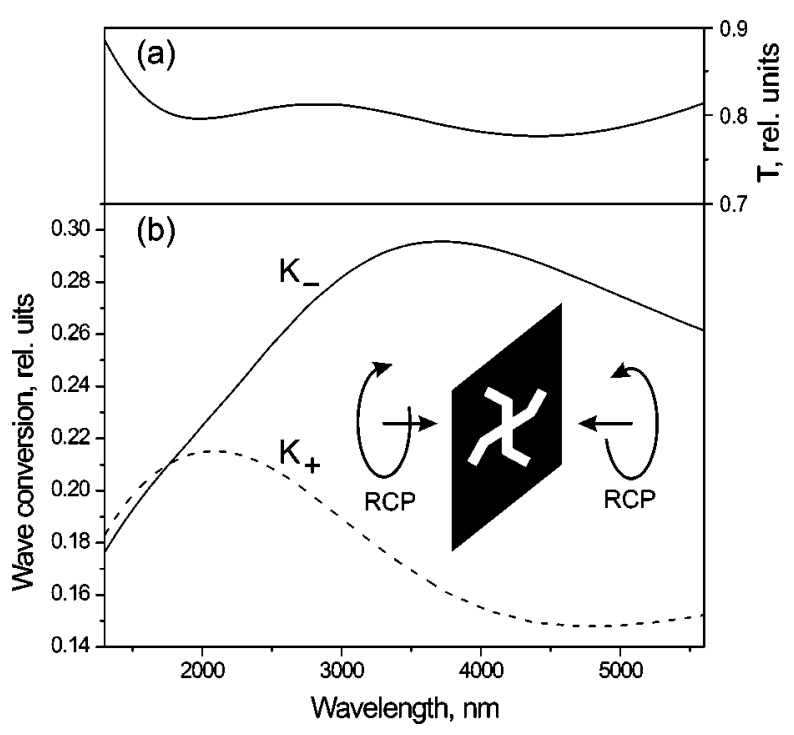

FIG. 2. (a) Wavelength dependence of the total transmission $T$ through the gammadion opening; (b) wavelength dependence of the total polarization conversion efficiencies $K_{ \pm}$for incident waves with the same (dashed line, $K_{+}$) and opposite (solid line, $K_{-}$) handedness of the gammadion. Inserted figure shows that the perceived sense of twist of a hole in a flat screen depends on from which side of the screen it is observed. The polarization conversion efficiency for a wave of a given handedness depends on whether it falls on the screen from one side or another. 


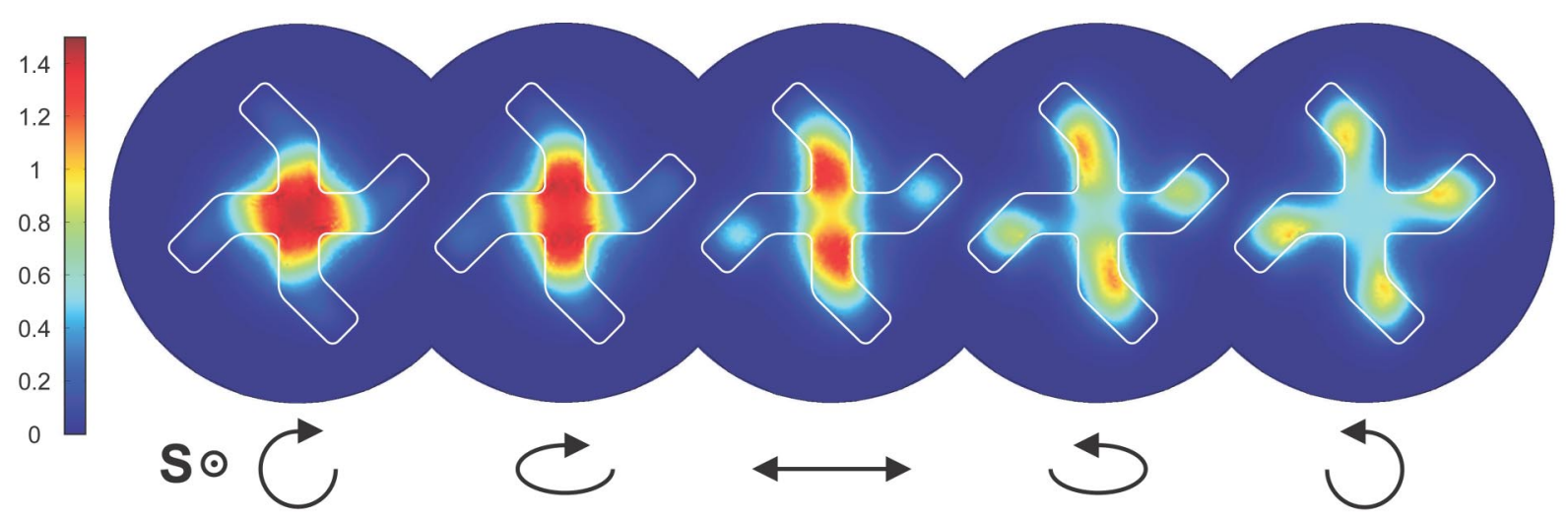

FIG. 3. (Color online) Dependence of the field structure behind the gammadion hole on the polarization state of incident light indicated by arrows.

depends on what side the screen is observed from, the polarization conversion efficiency depends on whether the light wave enters the screen from one side or the other (see the inset in Fig. 2).

We observed another interesting and potentially useful phenomenon which may be seen in Fig. 3. The light field behind the screen is strongly concentrated at the center of the gammadion when handedness of the chiral hole and the polarization state of the wave are the same. Here, light energy is concentrated in a spot with a diameter much smaller than both the wavelength and the characteristic size of the gammadion. In contrast, for opposite incident polarization no concentration effect is seen and the transmitted light energy is spread across the whole opening.

In summary, we have found polarization conversion and nanofocusing of circularly polarized light transmitted through a chiral hole in a metallic screen. Both depend on the mutual sense of twist of the hole and the handedness of the incident wave. They are pronounced for wavelengths close to the overall size of the opening and disappears for shorter wavelengths.
The authors acknowledge the support of the Engineering and Physical Sciences Research Council (UK) and fruitful discussions with A. Zayats.

${ }^{1}$ T. W. Ebbesen, H. J. Lezec, H. F. Ghaemi, T. Thio, and P. A. Wolff, Nature (London) 391, 667 (1998).

${ }^{2}$ F. G. Garcia de Abajo, Opt. Express 10, 1475 (2002).

${ }^{3}$ J. A. Matteo, D. P. Fromm, Y. Yuen, P. J. Schunk, W. E. Moerner, and L. Hesselink, Appl. Phys. Lett. 85, 648 (2004).

${ }^{4}$ J. Elliott, I. I. Smolyaninov, N. I. Zheludev, and A. V. Zayats, Opt. Lett. 29, 1414 (2004).

${ }^{5}$ A. Degiron, H. J. Lezec, N. Yamamoto, and T. W. Ebbesen, Opt. Commun. 239, 61 (2004).

${ }^{6}$ A. Papakostas, A. Potts, D. M. Bagnall, S. L. Prosvirnin, H. J. Coles, and N. I. Zheludev, Phys. Rev. Lett. 90, 107404 (2003).

${ }^{7}$ A. S. Schwanecke, A. Krasavin, D. M. Bagnall, A. Potts, A. V. Zayats, and N. I. Zheludev, Phys. Rev. Lett. 91, 247404 (2003).

${ }^{8}$ A. Potts, D. M. Bagnall, and N. I. Zheludev, J. Opt. A, Pure Appl. Opt. 6, 193 (2004).

${ }^{9}$ Handbook of Optical Constants of Solids edited by E. D. Palik (Academic, New York, 1984).

${ }^{10}$ J. Jin, The Finite Element Method in Electrodynamics (Wiley, New York, 2002).

${ }^{11}$ Y. P. Svirko and N. I. Zheludev, Polarization of Light in Nonlinear Optics (Wiley, Chichester, 1998).

${ }^{12}$ J. D. Jackson, Classical Electrodynamics (Wiley, New York, 1999).

${ }^{13}$ I. R. Hooper and J. R. Sanbles, Opt. Lett. 27, 2152 (2002). 